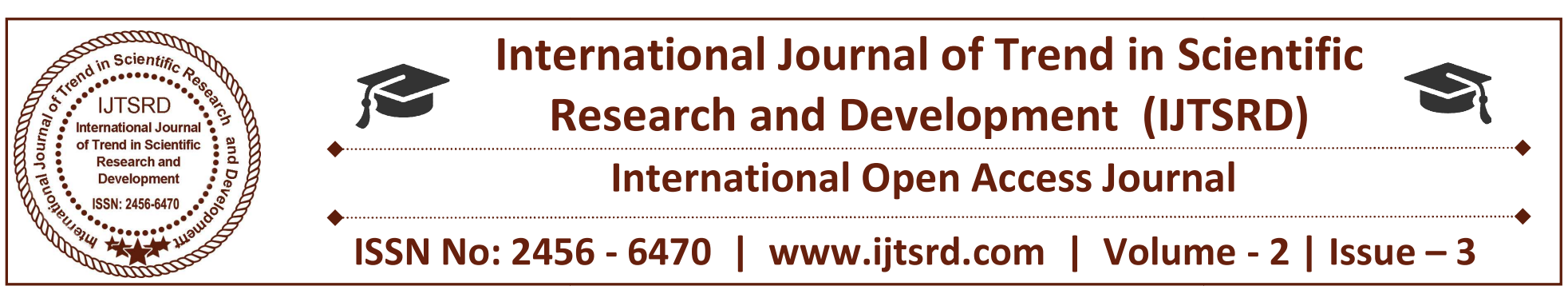

\title{
Genetic Programming Approach for Oral Cancer Detection and its Image Restoration
}

\author{
Rasika Joat \\ M.E. Student, Department of Electronics and \\ Telecommunication, Sipna COET, \\ Amravati, Maharashtra, India
}

\author{
Dr. A. P. Thakare \\ HOD, Department of Electronics and \\ Telecommunication, Sipna COET, \\ Amravati, Maharashtra, India
}

\begin{abstract}
Dr. Ketaki Kalele
Dr. Viashali Thakare

M.D.S, Assistant Professor,

V.Y.W.S Dental College \& Hospital,

Amravati, Maharashtra, India
\end{abstract}

\section{ABSTRACT}

Cancer is one of the leading causes of death in developing countries. Cancers are of different types like breast cancer, lung cancer, skin cancer and so on. Oral Cancer is one of the types of cancers. Oral cancer is a very common type of cancer. This Oral Cancer is observed in both males as well as females. It is a big challenge to detect Oral Cancer. This is a time consuming process in medical image processing. Detection and prevention of oral cancer at early stage is critical. But it increases the chances of survival. This work presents the detection of oral cancers using Image Processing. Clinical images which are RGB images and microscopic images are used as the input image for detection of cancer. At first, Gabor filter is used to remove noise from the images. This is used for image enhancement in image preprocessing step. Genetic Algorithm is used to extract the features of tumors from the enhanced image. GA is used for segmentation of image. The proposed algorithm provides better segmentation. Genetic Programming (GP), is a computation technique that can evolve better solutions for image classification problems.

Keywords: Oral Cancer, Clinical Images, Microscopic images, image enhancement, Gabor filter, Genetic Algorithm

\section{INTRODUCTION}

Cancer is a collection of related diseases. In all types of cancer, some cells of body begin to divide without stopping and spread into surrounding tissues. Human body is made up of trillions of cells and cancer can start almost anywhere in the human body. Human cells grow and divide. This is to form new cells as the body needs them. When cells grow old or becomes damaged, they die and new cells take their place. When cancer develops, however, this orderly process breaks down. As cells become more and more abnormal, old or damaged cells survive when they should die, and new cells form when they are not needed. These cells are not able to stop and continue to divide. This results in formation of tumors. Many cancers form solid tumors, which are masses of tissue.

Oral cancer is cancer that starts in the mouth or throat. Oral cancer is fairly common and very curable if found and treated at an early stage. More than $90 \%$ of all oral cavity tumors are squamous cell carcinoma, according to the National Cancer Institute. Squamous cells make up the lining of the oral cavity, also called the mucosa. As cancer in the lining of the mouth grows, it can spread deeper into the nearby tissues of the mouth. Verrucous carcinoma is another type of oral cancer. It's considered a type of squamous cell carcinoma, but this low-grade cancer rarely spreads to distant sites (metastasizes). It accounts for less than 
$5 \%$ of all diagnosed oral cancer. Other much less common types of oral cancer include tumors of the salivary glands, including adenoid cystic carcinoma, adenocarcinoma, and other types of salivary gland cancer. Oral tumors are mainly classified as Benign and Malignant. Benign tumors are noncancerous and they seldom grows back where as malignant tumors are cancerous and they rapidly grows and invade to the surrounding healthy tissue.The location of tumor helps the individual to determine how it effects an individual normal functioning.[6]

Systemic problems those that affect the entire body many times appear in the mouth first. In general, mouth is a good indicator of what's going on in the body, which is why the physicians for generations have asked patients to open their mouth. The discovery of a wound in the mouth indicates so many problems in the Human Body Despite advances in surgery, radiation and chemotherapy, the mortality rate associated with oral cancer has no improvement in the last 40 years. Eventually, 50 percent of people who have oral cancer die as a result of the malignancy. Early evaluation of oral precancerous lesions can have a dramatic impact on oral cancer mortality rates. Tumors can be benign, premalignant or malignant. Benign tumors are harmless and do not spread. Premalignant tumors can transform into Malignant. Malignant tumors are cancerous. Oral cancer can affect any area of the oral cavity including the lips, gum tissues, tongue, cheek lining and the hard and soft palate. - Maharashtra has the highest incidence of mouth cancer in the world. The common oral precancerous lesions are leukoplakia, erythroplakia, and oral sub - mucous fibrosis (OSF). The diagnosis of Oral pre-cancer and cancer remains a challenge to the dental profession, particularly in the detection, evaluation and management of early phase alterations or frank disease [7]. Prediction of Oral Leukoplakia (premalignant) and Oral Squamous Cell Carcinoma becomes a challenging task. Due to the lack of timely diagnosis, in all conventional methods or differential diagnosis, Biopsy is required [4].

This project focuses on detecting and classifying oral cancers at an earlier stage. $\mathrm{X}$ - Rays are an essential part of dental care. Although $\mathrm{X}$ - rays are effective diagnostic tools, some dental practices particularly those that handle a large number of dental implant cases, are using more advanced imaging techniques to ensure an even higher degree of accuracy. Dental radiographs are used for screening oral pathologies continuously and it is often a difficult task to detect early stage cancer tissues in a dental radiograph. Unlike other types of cancers, oral cancers are visibly seen with the naked eye, some cancers are located internally in the mouth, making their detection difficult. And also some non cancerous tissues are not harmful. The input clinical image is preprocessed using Gabor filter. Later, the image is segmented and the tumor is detected. The proposed technique will quantify each result with the diagnostic accuracy and helps the radiologist as a second guidance.

Image classification has gained immense importance in recent years as image processing and machine vision are widely used in daily life applications such as face recognition recognition, medical imaging systems and remote sensing. Genetic Programming (GP) is a method that provides solution to a user defined problem by evolving a computer program. It is inspired by the Darwinian Principle of natural selection that includes operators like selection, crossover and mutation to evolve diverse solutions for the problem. With the flexible representation, GP has provided promising solutions that hardly can be thought of by humans. GP has been extensively employed for pattern recognition, object detection and classification. Moreover, GP methods have been employed for various tasks such as feature extraction which is a process of transforming images into feature values, feature selection which selects good features among whole set of features, and feature construction which builds new features from existing feature set. Feature selection and construction aim at dimensionality reduction that reduces the search space for GP for evolving good solutions meanwhile speeding up the search process by using less number of features in the reduced search space [1].

\section{SYSTEM ARCHITECTURE}

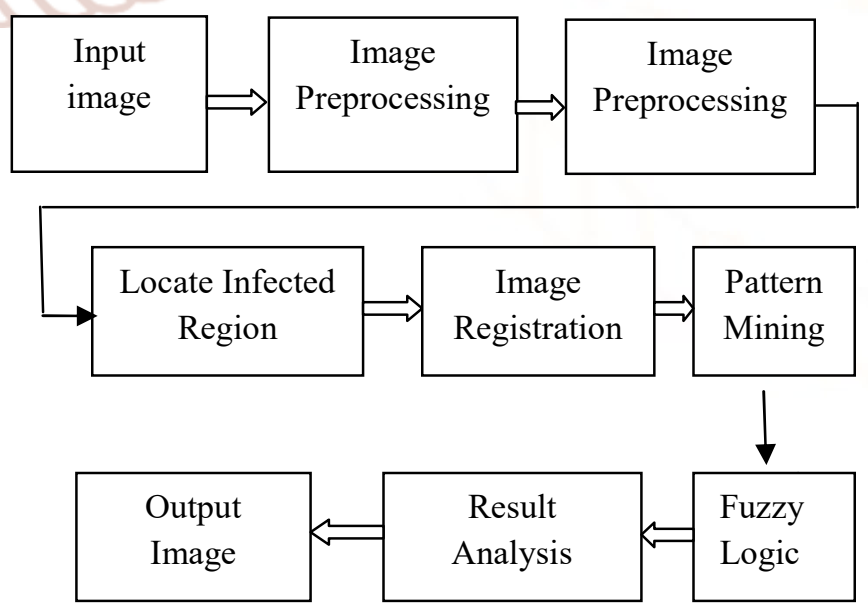

Fig 1 System block diagram 
This system uses genetic algorithm for feature extraction from input image An input image is RGB image of mouth or oral cavity.

Image Preprocessing- Image is given as an input to the Gabor filter. This is used for removing noise from an image. Gabor filter is a linear filter used for texture analysis. This means that it analyses whether there are any specific frequency content in the image in specific direction in a localized region.

Genetic Algorithm- Genetic Algorithm (GA) is a search-based optimization technique based on the principles of Genetics and Natural Selection. It is frequently used to find optimal or near-optimal solutions to difficult problems which otherwise would take a lifetime to solve. It is frequently used to solve optimization problems, in research, and in machine learning. Optimization refers to finding the values of inputs in such a way that we get the "best" output values. In GAs, we have a pool or a population of possible solutions to the given problem. These solutions then undergo recombination and mutation (like in natural genetics), producing new children, and the process is repeated over various generations. Each individual (or candidate solution) is assigned a fitness value (based on its objective function value) and the fitter individuals are given a higher chance to mate and yield more "fitter" individuals.

Locate infected region- The output image from genetic algorithm is then cropped. Only the infected region is the region of interest.

Image Registration- In image registration the input image is compared with image showing infection. The infected section is shown by green color in the input image.

Pattern Mining- In pattern mining, the pattern of infection found is compared with the patterns stored in the database. Pattern matching is done and the pattern which is perfectly matched with the input image infection is selected from the database.

Fuzzy Logic-Fuzzy rules are implemented to calculate the percentage of infection in an image. These fuzzy rules are useful in finding the stage of oral cancer. It provides the fuzzy conclusion about type of oral cancer.

Result Analysis-This is the final stage of system. In result analysis, two images are compared on the basis

of RGB histograms . Different parameters like signal to noise ratio, standard deviation are calculated.

\section{Algorithm}

- Input image is either clinical image or microscopic image. Input is taken from database.

- Image preprocessing is done on input image. This will removes noise.

- Segmentation is done with the help of genetic algorithm.

- Locate the infected region and image registration is carried out on image.

- Pattern mining is carried out to check which pattern in database is similar to the input image.

- Fuzzy logic is applied to check which patterns are matching with input and gives fuzzy conclusion.

- Result Analysis gives the comparison between two images on the basis of entropy, standard deviation, mean of images and RGB histogram.

\section{RESULT ANALYSIS}

There are clinical images as well as microscopic images taken from experts. Results are observed for both types of images.

\section{Clinical image as an input}

Figure 2 shows clinical input image. The image is preprocessed using Gabor filter and figure 3 shows the output of this stage. Figure 4 shows an image output of Genetic Algorithm stage. The region of interest is then acquired as shown in figure 5. The region of interest (ROI) is shown in figure with black section and white section is not required part of an image.

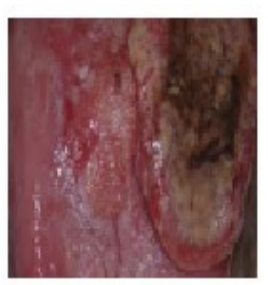

Fig 2 Input Image

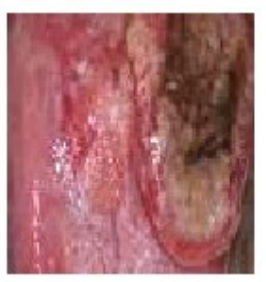

Fig 3 Preprocessed image 


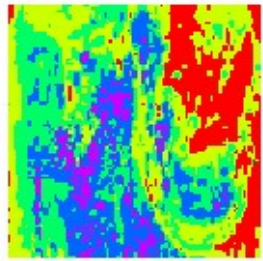

Fig 4 GA Output

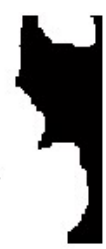

Fig 5 ROI

Image registration is the next step. In this step, the infection in the image is shown with respect to the original input image. This is shown in figure 6.

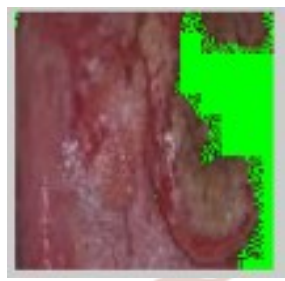

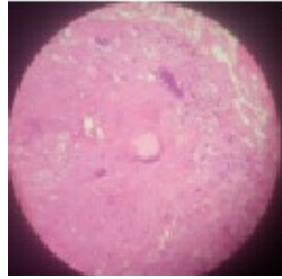

Fig 8 Input image

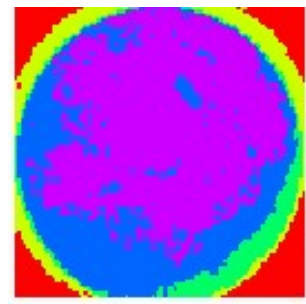

Fig 10 GA output

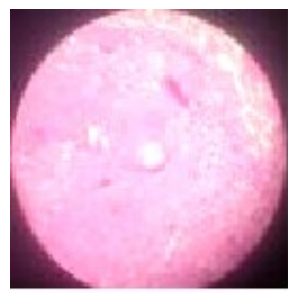

Fig 9 Preprocessed image

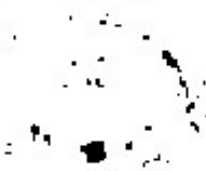

Fig 11 ROI

The above figure 8 is input microscopic image and figure 9 is preprocessed version of it. GA gives output in as shown in figure 10 and region of interest is Fig 6 Registered Image shown in figure 11 .

The parameters calculated are as follows:

\begin{tabular}{|c|c|}
\hline Total number of pixels & 10000 \\
\hline Infected pixels & 1695 \\
\hline Percentage of infection & $16.95 \%$ \\
\hline Infection level & Average \\
\hline Registration time(sec) & 0.1512 \\
\hline Infection time $(\mathrm{sec})$ & 0.4649 \\
\hline Mining time $(\mathrm{sec})$ & 3.2495 \\
\hline
\end{tabular}

$=$

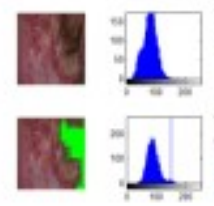

Fig 7 Result analysis

The above figure shows the comparison of input image and registered image with their RGB histograms respectively. The results observed are given below.

Mean Square error- 1662.4579

Peak Signal to Noise ratio-15.9233

Normalized cross correlation-1.1165

Microscopic image as an input

In this section input image is microscopic image.

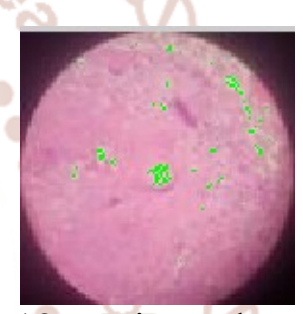

Fig 12 Registered Image

The above figure 12 shows registered image. Green portion in image shows infection.

\section{CONCLUSION}

Cancer classification and diagnosis is an emerging research area in the field of medical image processing. In this project genetic Programming is used as a feature extractor for solving user defined problem. There are total 25 images out of which 23 images gives desired results. Accuracy of this proposed work is $92 \%$. The level and percentage of infection is calculated.

\section{REFERENCES}

1) Qurrat Ul Ain, Bing Xue, Harith Al-Sahaf, and Mengjie Zhang, 'Genetic Programming for Skin Cancer Detection in Dermoscopic Images,' IEEE 978-1-5090-4601-0/17, 2017.

2) Rahul Kumar Singh, Sarif Kumar Naik2, Lalit Gupta2, Srinivasan Balakrishnan, Hybrid SVM Random Forest Classification System for Oral 
Cancer Screening using LIF Spectra,IEEE, 7) Narayan Naik, Anusha Amin, Dechamma K.C, Tampa, FL, USA, 1051-4651

3) Belvin Thomas, Dr.Vinod Kumar, Dr. Sunil Saini, Texture Analysis Based Segmentation and Classification of Oral Cancer Lesions in Color Images Using ANN, Signal Processing Computing and Control (ISPCC) 2013 IEEE International Conference on, pp. 1-5, 2013.

4) Ghassan Hamarneh, Artur Chodorowski, and Tomas Gustavssoni,Active Contour Models: Application to Oral Lesion Detection in Color Images, : Systems, Man, and Cybernetics, 2000 IEEE International Conference

5) Tanupriya Choudhury,Vivek Kumar, Darshika Nigam, Bhaskar Mandal, Intelligent Classification of Lung Oral Cancer through diverse data mining algorithms, Published in: Micro-Electronics and Telecommunication Engineering (ICMETE), 2016 International Conference.

6) M. Praveena Kiruba bai , A Survey on the Detection of Oral Cancer, published in International Journal of Innovations \& Advancement in Computer Science (IJIACS), 2017. Deepthi B.A, Nidhi Hegde, Oral Cancer Detection Using Android Application, published in International Journal of Advanced Research in Computer Science and Software Engineering, May 2017.

8) Yi-Ying Wang, Shao-Chien Chang, Li-Wha Wu, Sen-Tien Tsai and Yung-Nien Sun, A ColorBased Approach for Automated Segmentation in Tumor Tissue Classification, published in conference of IEEE 2007.

9) Rahul Kumar Singh, Sarif Kumar Naik, Lalit Gupta, Srinivasan Balakrishnan, Santhosh C and Keerthilatha M Pai , Hybrid SVM - Random Forest Classification System for Oral Cancer Screening using LIF Spectra, IEEE conference, 2008.

10) Anuradha.K, Dr. K. Sankaranarayanan, Statistical Feature Extraction to classify oral cancers, published in Journal of Global Research in Computer Science, 2013. 